\title{
A Weak Convergence to Hermite Process by Martingale Differences
}

\author{
Xichao Sun and Ronglong Cheng \\ Department of Mathematics and Physics, Bengbu College, 1866 Caoshan Road, Bengbu, Anhui 233030, China \\ Correspondence should be addressed to Xichao Sun; sunxichao626@126.com
}

Received 5 May 2014; Accepted 25 June 2014; Published 14 July 2014

Academic Editor: Carlo Cattani

Copyright ( 2014 X. Sun and R. Cheng. This is an open access article distributed under the Creative Commons Attribution License, which permits unrestricted use, distribution, and reproduction in any medium, provided the original work is properly cited.

We consider the weak convergence to general Hermite process $Z_{H, k}$ of order $k$ with index $H$. By applying martingale differences we construct a sequence $\left\{Z_{H, k}^{(n)}, n=1,2, \ldots\right\}$ of multiple Wiener-Itô stochastic integrals such that it converges in distribution to the Hermite process $Z_{H, k}$.

\section{Introduction}

The self-similar stochastic processes, which are exactly of approximately similar to a part of themselves, have been widely studied since their practical applications to internet traffic, hydrology, turbulence, and finance. We refer to the work of Taqqu [1] for a guide on the appearance of the self-similarity in many applications and to the monographs by Embrechts and Maejima [2] and by Samorodnitsky and Taqqu [3] for complete expositions on self-similar processes. In this paper, we consider the so-called Hermite process with index $H \in(1 / 2,1)$, which is a special class of self-similar processes with long range dependency. This process is given as limits of the so called Non Central Limit Theorem studied by Dobrushin and Major [4] and Taqqu [5].

Consider a stationary centered Gaussian sequence $\left(\xi_{n}\right)_{n \in(\mathbb{N})}$ with $E\left(\xi_{n}^{2}\right)=1$ such that

$$
r(n):=E\left(\xi_{0} \xi_{n}\right)=n^{(2 H-2) / k} L(n),
$$

where $k \geq 1$ are integers, $H \in(1 / 2,1)$, and $L$ is a slowly varying function at infinity. Denote by $H_{m}(x)$ the Hermite polynomial of degree $m$ defined by

$$
H_{m}(x)=(-1)^{m} e^{x^{2} / 2} \frac{d^{m}}{d x^{m}} e^{-x^{2} / 2}, \quad m=1,2, \ldots
$$

and $H_{0}(x)=1$. Let the function $g: \mathbb{R} \rightarrow \mathbb{R}$ satisfy $E\left(g\left(\xi_{0}\right)\right)=0$ and $E\left(g\left(\xi_{0}\right)^{2}\right)<\infty$. Suppose that $g$ has Hermite rank equal to $k$, that is, if $g$ admits the following expansion in Hermite polynomials:

$$
g(x)=\sum_{j=0}^{\infty} c_{j} H_{j}(x), \quad c_{j}=\frac{1}{j !} E\left(g\left(\xi_{0} H_{j}\left(\xi_{0}\right)\right)\right) .
$$

The Hermite rank of $g$ is defined by

$$
k=\min \left\{j ; c_{j} \neq 0\right\} .
$$

Since

$$
E\left[g\left(\xi_{0}\right)\right]=0,
$$

we have $k \geq 1$. Then, the Non Central Limit Theorem (see $[4,5])$ implies that the sequence of stochastic processes of the form

$$
\frac{1}{n^{H}} \sum_{j=1}^{[n t]} g\left(\xi_{j}\right), \quad n=1,2, \ldots
$$

converges, as $n \rightarrow \infty$, in the sense of finite dimensional distributions to the process

$$
\begin{aligned}
& Z_{H, k}(t) \\
& \quad=\int_{[0, t]^{k}} Q\left(t, s_{1}, \ldots, s_{k}\right) d B\left(s_{1}\right) \cdots d B\left(s_{k}\right), \quad t \in[0,1],
\end{aligned}
$$


where the integral is a multiple Wiener-Itô stochastic integral with respect to standard Brownian motion $B=\{B(t), t \geq 0\}$ and

$$
\begin{aligned}
& Q\left(t, s_{1}, \ldots, s_{k}\right) \\
& =c(H, k)\left(\prod_{j=1}^{k} s_{j}^{(1 / 2)-H_{0}}\right) \\
& \quad \times \int_{0}^{t} u^{k\left(H_{0}-(1 / 2)\right)} \prod_{j=1}^{k}\left(u-s_{j}\right)_{+}^{H_{0}-(3 / 2)} d u
\end{aligned}
$$

with $H_{0}=1-((1-H) / k) \in(1-(1 / 2 k), 1), s_{1}, \ldots, s_{k} \in$ $[0, t]$ and a positive normal constant $c(H, k)$ such that $E\left[\left(Z_{H, k}(1)\right)^{2}\right]=1$.

Definition 1 (see [5]). Let $k \geq 1$ be integer and let $H \in$ $(1 / 2,1)$. The process $\left(Z_{H, k}(t)\right)_{t \geq 0}$ defined by (7) is called the Hermite process of order $k$, with index $H$.

Clearly, when $k=1$ the Hermite process is the fractional Brownian motion with Hurst parameter $H \in(1 / 2,1)$. When $k=2$ the Hermite process is called the Rosenblatt process (see [6]). It is important to note that the Hermite process is not Gaussian for $k \geq 2$. The simplest Hermite process is the fractional Brownian motion. The Rosenblatt process is the simplest non-Gaussian Hermite process. More work for the Hermite process and related processes can be found in Bardet and Tudor [7], Chronopoulou et al. [8], Li [9, 10], Li and Lim [11], Heydari et al. [12, 13], Maejima and Tudor [14], Pipiras and Taqqu [15], Torres and Tudor [16], Tudor [17], Tudor and Viens [18], and the references therein. The Hermite processes are neither a semimartingale nor a Markov process, and they admit the same properties as Gaussian fractional Brownian motion. We mention the following:

(i) it exhibits long-range dependence in the sense that

$$
\sum_{n \geq \alpha} \rho_{n}(\alpha)=\infty
$$

for any $\alpha>0$, where

$$
\begin{aligned}
& \rho_{n}(\alpha) \\
& \quad=E\left[\left(Z_{H, k}(\alpha+1)-Z_{H, k}(\alpha)\right)\left(Z_{H, k}(n+1)-Z_{H, k}(n)\right)\right] .
\end{aligned}
$$

(ii) it is $H$-selfsimilar in the sense that for any $c>0$,

$$
\left\{Z_{H, k}(c t), t \geq 0\right\} \stackrel{d}{=}\left\{c^{H} Z_{H, k}(t), t \geq 0\right\},
$$

where $\stackrel{d}{=}$ indicates here equality of the finitedimensional distributions;

(iii) it has stationary increments, that is, the joint distribution of $\left(Z_{H, k}(t+h)-Z_{H, k}(t), h \geq 0\right)$ is independent of every $t \geq 0$; (iv) its covariance function is

$$
E\left[Z_{H, k}(t) Z_{H, k}(s)\right]=\frac{1}{2}\left[t^{2 H}+s^{2 H}-|t-s|^{2 H}\right]
$$

(v) it is Hölder continuous of order $\gamma<H$.

These properties of the Hermite process motivate our interesting to study them. Among the applications of the Hermite processes in statistics or econometrics, for example, see Hall et al. [19], Leonenko and Woyczynski [20], and Tudor [17]. Besides these more or less practical applications of the Hermite processes, our motivation is also theoretical; it comes from the recent intensive interest to push further the stochastic calculus with respect to more and more general integrator processes. We believe that such class of processes constitutes an interesting and instructive example where the recent developed techniques of the generalized stochastic calculus can find a significant test bench. On the other hand, the fractional Brownian motion has become an object of intense study in recent some years, due to its interesting properties and its applications in various scientific areas including telecommunications, turbulence, image processing, and finance. Some surveys and complete literature for fractional Brownian motion could be found in Alós et al. [21], Biagini et al. [22], $\mathrm{Hu}$ [23], Mishura [24], Nieminen [25], Nualart [26], and the references therein. However, in contrast to the extensive studies on fractional Brownian motion, there has been little systematic investigation on the general Hermite process. The main reasons for this, in our opinion, are the complexity of dependence structures and property of non-Gaussian. Therefore, it seems interesting to study the Hermite process. In this short paper we will prove a weak convergence theorem to the Hermite process based on martingale differences. Our main object is to explain and prove the following theorem.

Theorem 2. Let $\left(\xi^{(n)}\right)_{n \geq 1}$ be a sequence of square integrable martingale differences satisfying the following conditions:

$$
\lim _{n \rightarrow \infty} \frac{\left(\xi_{i}^{(n)}\right)^{2}}{1 / n}=1 \quad \text { a.s. }
$$

for every $i \geq 1$ and

$$
\max _{1 \leq i \leq n}\left|\xi_{i}^{(n)}\right| \leq \frac{C}{\sqrt{n}} \quad \text { a.s. }
$$

for some $C \geq 1$. Define the two sequences $\left\{W^{n}\right\}$ and $\left\{Z^{n}\right\}$ of processes as follows:

$$
\begin{gathered}
W_{t}^{n}:=\sum_{i=1}^{[n t]} \xi_{i}^{(n)}, \quad 0 \leq t \leq 1, \\
Z_{t}^{n}:=\int_{\mathbb{R}^{k}} Q^{n}\left(t, s_{1}, \ldots, s_{k}\right) d W_{s_{1}}^{n} \cdots d W_{s_{k}}^{n}, \quad 0 \leq t \leq 1,
\end{gathered}
$$


where $Q^{n}\left(t, s_{1}, \ldots, s_{k}\right)$ is given by

$$
\begin{aligned}
& Q^{n}\left(t, s_{1}, \ldots, s_{k}\right) \\
& =c(H, k) n^{k} \\
& \quad \times \int_{\left(\left(s_{1}-1\right) / n\right)}^{\left(s_{1} / n\right)} \cdots \int_{\left(\left(s_{k}-1\right) / n\right)}^{\left(s_{k} / n\right)} Q\left(\frac{[n t]}{n}, r_{1}, \ldots, r_{k}\right) \\
&
\end{aligned}
$$

with $s_{1}, \ldots, s_{k} \in[1 / n, 1]$. Then the process $Z^{n}$ converges in distribution to the Hermite process $Z_{H, k}$ as $n$ tends to infinity.

Remark 3. One eliminates the diagonal " $s_{1} \neq \cdots \neq s_{k}$ " because the Hermite process is defined as a multiple Wiener-Itô integral as a consequence it has zero mean.

This paper is organized as follows. In Section 2 we present some preliminaries for the Hermite process. In Section 3 we will give the proof of Theorem 2 .

\section{Hermite Processes}

In this section, we briefly recall some basic properties of the Hermite process. We refer to Maejima and Tudor [14], Peccati and Taqqu [27], and Pipiras and Taqqu [15] for some complete descriptions of the Hermite processes. For simplicity we let $C$ stand for a positive constant depending only on subscripts and its value may be different in different appearances. Throughout this paper we assume that $1 / 2<$ $H<1$, and let $Z_{H, k}=\left\{Z_{H, k}(t)\right\}_{t \in \mathbb{R}}$ be a Hermite process of order $k$ with index $H \in(1 / 2,1)$. As we pointed out before, we shall use Hermite processes to illustrate the preceding discussion. The Hermite processes $\left\{Z_{H, k}(t), t \geq 0\right\}$ are selfsimilar processes with parameter $H \in(1 / 2,1)$, that is, for any $c>0$,

$$
\left\{Z_{H, k}(c t), t \geq 0\right\} \stackrel{d}{=}\left\{c^{H} Z_{H, k}(t), t \geq 0\right\}
$$

where $\stackrel{d}{=}$ indicates here equality of the finite-dimensional distributions. They have also stationary increments and are represented by multiple integrals of order $k$ and hence are non-Gaussian when $k \geq 2$. The simplest Hermite process is fractional Brownian motion. The Rosenblatt process is the simplest non-Gaussian Hermite process. The Hermite processes are neither a semimartingale nor a Markov process. They have the four representations, which are as follows (see $[15,27])$ :

(i) time domain representation: for $t \in \mathbb{R}$

$$
Z_{H, k}(t)=\int_{\mathbb{R}^{k}} f\left(t, s_{1}, \ldots, s_{k}\right) d B\left(s_{1}\right) \cdots d B\left(s_{k}\right),
$$

where the integral is multiple Wiener-Itô integral of order $k$ with respect to the standard Brownian motion $B((t))_{t \in \mathbb{R}}$ and the kernel $f$ is defined as

$$
f\left(t, s_{1}, \ldots, s_{k}\right):=c(H, k) \int_{0}^{t}\left(\prod_{j=1}^{k}\left(u-s_{j}\right)_{+}^{H_{0}-(3 / 2)}\right) d u,
$$

with $H_{0}=1-((1-H) / k) \in(1-(1 / 2 k), 1), s_{1}, \ldots, s_{k} \in$ $\mathbb{R}, x_{+}=\max \{x, 0\}$, and

$$
\begin{aligned}
& c(H, k) \\
& =\left(\frac{H(2 H-1)[\Gamma((1 / 2)+((1-H) / k))]^{k}}{k ![\Gamma((1 / 2)-((1-H) / k)) \Gamma(2(1-H) / k)]^{k}}\right)^{1 / 2} ;
\end{aligned}
$$

(ii) positive half-axis representation: for $t \geq 0$

$$
Z_{H, k}(t)=\int_{[0, \infty)^{k}} g\left(t, s_{1}, \ldots, s_{k}\right) d B\left(s_{1}\right) \cdots d B\left(s_{k}\right),
$$

where the kernel $g$ is defined as

$$
\begin{aligned}
& g\left(t, s_{1}, \ldots, s_{k}\right) \\
& :=c(H, k) \int_{0}^{t}\left(\prod_{j=1}^{k} s_{j}^{(1 / 2)-H_{0}}\left(1-u s_{j}\right)_{+}^{H_{0}-(3 / 2)}\right) d u
\end{aligned}
$$

with $s_{1}, \ldots s_{k} \in \mathbb{R}^{+}$;

(iii) spectral domain representation: for $t \in \mathbb{R}$

$$
\begin{aligned}
& Z_{H, k}(t) \\
& =\widehat{c}(H, k) \int_{\mathbb{R}^{d}} \frac{e^{i\left(\lambda_{1}+\cdots+\lambda_{k}\right) t}-1}{i\left(\lambda_{1}+\cdots+\lambda_{k}\right)} \prod_{j=1}^{k}\left|\lambda_{j}\right|^{(1 / 2)-H_{0}} \\
& \quad \times d \widehat{W}\left(\lambda_{1}\right) \cdots d \widehat{W}\left(\lambda_{k}\right),
\end{aligned}
$$

where $\widehat{W}$ is a suitable complex-valued Brownian motion on $\mathbb{R}$ and

$$
\begin{aligned}
& \widehat{c}(H, k) \\
& =(k H(2 H-1) \\
& \quad \times\left(k ! \left[2 \Gamma\left(\frac{2(1-H)}{k}\right)\right.\right. \\
& \left.\left.\left.\quad \times \sin \left(\left(\frac{1}{2}-\frac{1-H}{k}\right) \pi\right)\right]^{k}\right)^{-1}\right)^{1 / 2} ;
\end{aligned}
$$

(iv) finite time interval representation: for $t \geq 0$,

$Z_{H, k}(t)$

$$
=\int_{[0, t]^{k}} Q\left(t, s_{1}, \ldots, s_{k}\right) d B\left(s_{1}\right) \cdots d B\left(s_{k}\right), \quad t \in[0,1],
$$


where the integral is a multiple Wiener-Itô stochastic integral with respect to standard Brownian motion $B=\{B(t), t \geq 0\}$ and

$$
\begin{aligned}
Q\left(t, s_{1}, \ldots, s_{k}\right)= & c(H, k)\left(\prod_{j=1}^{k} s_{j}^{(1 / 2)-H_{0}}\right) \\
& \times \int_{0}^{t} u^{k\left(H_{0}-(1 / 2)\right)} \prod_{j=1}^{k}\left(u-s_{j}\right)_{+}^{H_{0}-(3 / 2)} d u
\end{aligned}
$$

with $s_{1}, \ldots s_{k} \in[0, t]$.

\section{Proof of Theorem 2}

For simplicity we let $C$ stand for a positive constant depending only on subscripts and its value may be different in different appearances. Denote by $D=D(0,1)$ the Skorohod space of right continuous functions on the interval $[0,1]$ with left-hand limits and equip $D$ with the metric

$$
\begin{array}{r}
d(u, v):=\inf \{\varepsilon>0: \exists \lambda \in \Lambda \text { such that }\|\lambda\| \leq \varepsilon \\
\text { and } \left.\sup _{t}|u(t)-v(\lambda(t))| \leq \varepsilon\right\},
\end{array}
$$

where $\|\lambda\|:=\sup _{s \neq t}|\log (\lambda(t)-\lambda(s)) /(t-s)|$ and

$$
\Lambda:=\{\lambda:[0,1] \longrightarrow[0,1]
$$

a strictly increasing and continuous mapping .

Under this metric, $D$ is a separable and complete metric space. Let $X, X^{n}: \Omega \rightarrow D$ be random variables and

$$
T_{X}:=\left\{t \in(0,1): P\left(X_{t} \neq X_{t-}\right)=0\right\} \cup\{0,1\} .
$$

Recall that $X^{n}$ converges in distribution to $X$ if, for every bounded and continuous $\phi: D \rightarrow \mathbb{R}$,

$$
E\left(\phi\left(X^{n}\right)\right) \longrightarrow E(\phi(X))
$$

as $n$ tends to infinity. In order to prove Theorem 2 we need the three basic results. The first one is in Billingsley [28] which basically states that weak convergence in $D$ can be proved by using the convergence of finite-dimensional distributions and the tightness of a sequence.

Theorem 4. Suppose that $X, X^{n}: \Omega \rightarrow D$ are random variables and

$$
\left(X_{t_{1}}^{n}, \ldots, X_{t_{k}}^{n}\right) \stackrel{d}{\rightarrow}\left(X_{t_{1}}, \ldots, X_{t_{k}}\right)
$$

holds whenever $t_{1}, \ldots, t_{k} \in T_{X}$, where $\stackrel{d}{\rightarrow}$ means standard convergence in distribution. Assume that $P\left\{X_{1} \neq X_{1-}\right\}=0$ and that

$$
E\left\{\left|X_{t}^{n}-X_{t_{1}}^{n}\right|^{C}\left|X_{t_{2}}^{n}-X_{t}^{n}\right|^{C}\right\} \leq\left[F\left(t_{2}\right)-F\left(t_{1}\right)\right]^{2 \alpha}
$$

for $t_{1} \leq t \leq t_{2}$ and $n \geq 1$, where $C>0, \alpha>(1 / 2)$ and $F$ is a nondecreasing, continuous function on $[0,1]$. Then

$$
X^{n} \stackrel{d}{\rightarrow} X
$$

in the sense of (30).

Secondly, we need the Lindeberg condition (see [29]).

Theorem 5. Let $t \in(0,1], \sigma_{t}^{2} \geq 0$ and let $\left(\xi^{(n)}\right)_{n \geq 1}$ be a sequence of square integrable martingale differences satisfying the Lindeberg condition:

$$
\sum_{i=1}^{[n t]} E\left[\left(\xi_{i}^{(n)}\right)^{2} I_{\left\{\left|\xi_{i}^{(n)}\right|>\varepsilon\right\}} \mid \mathscr{F}_{i-1}^{n}\right] \stackrel{P}{\rightarrow} 0
$$

for $\varepsilon>0$. Then $\sum_{i=1}^{[n t]}\left(\xi_{i}^{(n)}\right)^{2} \stackrel{P}{\rightarrow} \sigma_{t}^{2}$ implies that the convergence

$$
W_{t}^{n}:=\sum_{i=1}^{[n t]} \xi_{i}^{(n)} \stackrel{d}{\rightarrow} \mathcal{N} \sim N\left(0, \sigma_{t}^{2}\right)
$$

holds, as $n$ tends to infinity.

Thirdly, we need the following auxiliary lemma.

Lemma 6. Let $Q$ be as in (8) and Let $\left(\xi^{(n)}\right)_{n \geq 1}$ satisfy conditions (13) and (14). Then, as $n$ tends to infinity

$$
\begin{aligned}
& \sum_{i_{1}, \ldots, i_{k}=1, i_{1} \neq \cdots \neq i_{k}}^{n} n^{2 k} \int_{\left(\left(i_{1}-1\right) / n\right)}^{\left(i_{1} / n\right)} \\
\cdots & \int_{\left(\left(i_{k}-1\right) / n\right)}^{\left(i_{k} / n\right)} Q\left(\frac{\left[n t_{u}\right]}{n}, s_{1}, \ldots, s_{k}\right) d s_{1} \cdots d s_{k} \\
\times & \int_{\left(\left(i_{1}-1\right) / n\right)}^{\left(i_{1} / n\right)} \cdots \int_{\left(\left(i_{k}-1\right) / n\right)}^{\left(i_{k} / n\right)} Q\left(\frac{\left[n t_{v}\right]}{n}, s_{1}, \ldots, s_{k}\right) \\
\longrightarrow & \int_{0}^{1} \ldots \int_{0}^{1} Q\left(t_{u}, s_{1}, \ldots, s_{k}\right) Q\left(t_{v}, s_{1}, \ldots, s_{k}\right) d s_{1} \ldots d s_{k}\left(\xi_{i_{1}}^{(n)} \ldots \xi_{i_{k}}^{(n)}\right)^{2}
\end{aligned}
$$

holds almost surely, for $t_{u}, t_{v} \in[0,1]$.

Proof. Let us take $t_{u}=t_{v}$ and prove the first the case, that is,

$$
\begin{aligned}
& \sum_{i_{1}, \ldots, i_{k}=1, i_{1} \neq \cdots \neq i_{k}}^{n} n^{2 k}\left(\int_{\left(\left(i_{1}-1\right) / n\right)}^{\left(i_{1} / n\right)}\right. \\
& \left.\quad \ldots \int_{\left(\left(i_{k}-1\right) / n\right)}^{\left(i_{k} / n\right)} Q\left(t_{u}, s_{1}, \ldots, s_{k}\right) d s_{1} \cdots d s_{k}\right)^{2} \\
& \times\left(\xi_{i_{1}}^{(n)} \ldots \xi_{i_{k}}^{(n)}\right)^{2} \\
& \longrightarrow \int_{0}^{1} \cdots \int_{0}^{1} Q^{2}\left(t_{u}, s_{1}, \ldots, s_{k}\right) d s_{1} \cdots d s_{k},
\end{aligned}
$$


holds a.s. as $n \rightarrow \infty$. For every $t_{1}, \ldots, t_{k} \in[0,1]$, define

$$
\begin{aligned}
& g_{n}\left(t_{1}, \ldots, t_{k}\right):=n^{k} \\
& \quad \times \sum_{i_{1}, \ldots, i_{k}=1, i_{1} \neq \cdots \neq i_{k}}^{n} I_{\left(\left(\left(i_{1}-1\right) / n\right),\left(i_{1} / n\right)\right]}\left(t_{1}\right) \cdots I_{\left(\left(\left(i_{k}-1\right) / n\right),\left(i_{k} / n\right)\right]}\left(t_{k}\right) \\
& \quad \times \int_{\left(\left(i_{1}-1\right) / n\right)}^{\left(i_{1} / n\right)} \cdots \int_{\left(\left(i_{k}-1\right) / n\right)}^{\left(i_{k} / n\right)} Q\left(t_{u}, s_{1}, \ldots, s_{k}\right) d s_{1} \cdots d s_{k} \\
& \quad \times\left(\frac{\xi_{i_{1}}^{(n)}}{1 / \sqrt{n}}\right) \cdots\left(\frac{\xi_{i_{k}}^{(n)}}{1 / \sqrt{n}}\right) .
\end{aligned}
$$

Then one has

$$
\begin{aligned}
& g_{n}^{2}\left(t_{1}, \ldots, t_{k}\right) \\
& =\left(n^{k} \int_{\left(\left(i_{1}-1\right) / n\right)}^{\left(i_{1} / n\right)} \cdots \int_{\left(\left(i_{k}-1\right) / n\right)}^{\left(i_{k} / n\right)} Q\left(t_{u}, s_{1}, \ldots, s_{k}\right) d s_{1} \cdots d s_{k}\right)^{2} \\
& \quad \times\left(\frac{\xi_{i_{1}}^{(n)}}{1 / \sqrt{n}}\right)^{2} \cdots\left(\frac{\xi_{i_{k}}^{(n)}}{1 / \sqrt{n}}\right)^{2},
\end{aligned}
$$

for $t_{j} \in\left(\left(i_{j}-1\right) / n, i_{j} / n\right], j=1, \ldots, k$ and

$$
\begin{aligned}
& \int_{0}^{1} \cdots \int_{0}^{1} g_{n}^{2}\left(s_{1}, \ldots, s_{k}\right) d s_{1} \cdots d s_{k} \\
& =\sum_{i_{1}, \ldots, i_{k}=1, i_{1} \neq \cdots \neq i_{k}}^{n} n^{2 k} \\
& \quad \times\left(\int_{\left(\left(i_{1}-1\right) / n\right)}^{\left(i_{1} / n\right)} \cdots \int_{\left(\left(i_{k}-1\right) / n\right)}^{\left(i_{k} / n\right)} \mathrm{Q}\left(t_{u}, s_{1}, \ldots, s_{k}\right) d s_{1} \cdots d s_{k}\right)^{2} \\
& \quad \times\left(\xi_{i_{1}}^{(n)} \ldots \xi_{i_{k}}^{(n)}\right)^{2} .
\end{aligned}
$$

Firstly, we prove that $g_{n}^{2}\left(t_{1}, \ldots, t_{k}\right)$ is a.s. uniformly integrable. In fact, together with Hölder's inequality, condition (14), and Fubini theorem, we get

$$
\begin{aligned}
& \int_{0}^{1} \cdots \int_{0}^{1} g_{n}^{2}\left(s_{1}, \ldots, s_{k}\right) d s_{1} \cdots d s_{k} \\
& =\sum_{i_{1}, \ldots, i_{k}=1, i_{1} \neq \cdots \neq i_{k}}^{n} n^{2 k}\left(\xi_{i_{1}}^{(n)} \cdots \xi_{i_{k}}^{(n)}\right)^{2} \\
& \quad \times\left(\int_{\left(\left(i_{1}-1\right) / n\right)}^{\left.i_{1} / n\right)} \cdots \int_{\left(\left(i_{k}-1\right) / n\right)}^{\left(i_{k} / n\right)} Q\left(t_{u}, s_{1}, \ldots, s_{k}\right) d s_{1} \cdots d s_{k}\right)^{2} \\
& \leq C \sum_{i_{1}, \ldots, i_{k}=1, i_{1} \neq \cdots \neq i_{k}}^{n} \int_{\left(\left(i_{1}-1\right) / n\right)}^{\left(i_{1} / n\right)} \ldots \int_{\left(\left(i_{k}-1\right) / n\right)}^{\left(i_{k} / n\right)} Q^{2}\left(t_{u}, s_{1}, \ldots, s_{k}\right) \\
& \times d s_{1} \cdots d s_{k}
\end{aligned}
$$

$$
\begin{aligned}
& \leq C \int_{[0,1]^{k}}\left(\prod_{j=1}^{k} s_{j}^{1-2 H_{0}} \int_{0}^{t_{u}} \int_{0}^{t_{u}} x^{k\left(H_{0}-(1 / 2)\right)} y^{k\left(H_{0}-(1 / 2)\right)}\right. \\
& \left.\quad \times \prod_{j=1}^{k}\left(x-s_{j}\right)_{+}^{H_{0}-(3 / 2)}\left(y-s_{j}\right)_{+}^{H_{0}-(3 / 2)} d x d y\right) \\
& \times d s_{1} \cdots d s_{k} \\
& =C \int_{0}^{t_{u}} \int_{0}^{t_{u}} x^{k\left(H_{0}-(1 / 2)\right)} y^{k\left(H_{0}-(1 / 2)\right)} \\
& \quad \times\left[\int_{[0,1]^{k}} \prod_{j=1}^{k} s_{j}^{1-2 H_{0}}\left(x-s_{j}\right)_{+}^{H_{0}-(3 / 2)}\left(y-s_{j}\right)_{+}^{H_{0}-(3 / 2)}\right.
\end{aligned}
$$

$$
\begin{aligned}
& \left.x d s_{1} \cdots d s_{k}\right] d x d y \\
& =C \int_{0}^{t_{u}} \int_{0}^{t_{u}} x^{k\left(H_{0}-(1 / 2)\right)} y^{k\left(H_{0}-(1 / 2)\right)} \\
& \quad \times\left[\int_{0}^{1} s^{1-2 H_{0}}(x-s)_{+}^{H_{0}-(3 / 2)}(y-s)_{+}^{H_{0}-(3 / 2)} d s\right]^{k} d x d y \\
& \leq C \int_{0}^{t_{u}} \int_{0}^{t_{u}}|x-y|^{\left(2 H_{0}-2\right) k} d x d y \\
& =C \int_{0}^{t_{u}} \int_{0}^{t_{u}}|x-y|^{2 H-2} d x d y \\
& \leq C \int_{0}^{1} \int_{0}^{1}|x-y|^{2 H-2} d x d y<\infty,
\end{aligned}
$$

since $1 / 2<H<1$, where we have used the fact

$$
\begin{aligned}
\int_{0}^{x \wedge y} s^{1-2 H_{0}}(x-s)_{+}^{H_{0}-(3 / 2)}(y-s)_{+}^{H_{0}-(3 / 2)} d s \\
\quad=\frac{\beta\left(2-2 H_{0}, H_{0}-(1 / 2)\right)}{(x y)^{H_{0}-(1 / 2)}}|x-y|^{2 H_{0}-2} .
\end{aligned}
$$

In addition, for $t_{j} \in\left(\left(i_{j}-1\right) / n, i_{j} / n\right], j=1, \ldots, k$,

$$
\begin{aligned}
& g_{n}^{2}\left(t_{1}, \ldots t_{k}\right) \\
& =\left(n^{k} \int_{\left(\left(i_{1}-1\right) / n\right)}^{\left(i_{1} / n\right)} \cdots \int_{\left(\left(i_{k}-1\right) / n\right)}^{\left(i_{k} / n\right)} Q\left(t_{u}, s_{1}, \ldots, s_{k}\right) d s_{1} \cdots d s_{k}\right)^{2} \\
& \quad \times\left(\frac{\xi_{i_{1}}^{(n)}}{1 / \sqrt{n}}\right)^{2} \cdots\left(\frac{\xi_{i_{k}}^{(n)}}{1 / \sqrt{n}}\right)^{2} \\
& \longrightarrow Q^{2}\left(t_{u}, s_{1}, \ldots, s_{k}\right)
\end{aligned}
$$


holds a.s., as $n$ tends to infinity, because

$$
\begin{aligned}
n^{k} \int_{\left(\left(i_{1}-1\right) / n\right)}^{\left(i_{1} / n\right)} \cdots \int_{\left(\left(i_{k}-1\right) / n\right)}^{\left(i_{k} / n\right)} Q\left(t_{u}, s_{1}, \ldots, s_{k}\right) d s_{1} \cdots d s_{k} \\
\quad \longrightarrow Q\left(t_{u}, s_{1}, \ldots, s_{k}\right), \\
\quad\left(\frac{\xi_{i_{j}}^{(n)}}{1 / \sqrt{n}}\right)^{2} \longrightarrow 1, \quad j=1,2, \ldots, k
\end{aligned}
$$

holds a.s., as $n$ tends to infinity. Thus

$$
\begin{aligned}
& \int_{0}^{1} \cdots \int_{0}^{1} g_{n}^{2}\left(s_{1}, \ldots, s_{k}\right) d s_{1} \cdots d s_{k} \\
& \quad \longrightarrow \int_{0}^{1} \cdots \int_{0}^{1} Q^{2}\left(t_{u}, s_{1}, \ldots, s_{k}\right) d s_{1} \cdots d s_{k}
\end{aligned}
$$

holds a.s., as $n$ tends to infinity, and (37) follows.

Next we will prove the original claim. Denote that $t_{u}^{n}:=$ $\left[n t_{u}\right] / n$ and $t_{v}^{n}:=\left[n t_{v}\right] / n$. From the previous discussion we know that when $n \rightarrow \infty$,

$$
\begin{aligned}
& \sum_{i_{1}, \ldots, i_{k}=1, i_{1} \neq \cdots \neq i_{k}}^{n} n^{2 k} \int_{\left(\left(i_{1}-1\right) / n\right)}^{\left(i_{1} / n\right)} \\
& \ldots \int_{\left(\left(i_{k}-1\right) / n\right)}^{\left(i_{k} / n\right)} Q\left(t_{u}, s_{1}, \ldots, s_{k}\right) d s_{1} \cdots d s_{k} \\
\times & \int_{\left(\left(i_{1}-1\right) / n\right)}^{\left(i_{1} / n\right)} \cdots \int_{\left(\left(i_{k}-1\right) / n\right)}^{\left(i_{k} / n\right)} Q\left(t_{v}, s_{1}, \ldots, s_{k}\right) d s_{1} \cdots d s_{k} \\
\times & \left(\xi_{i_{1}}^{(n)} \ldots \xi_{i_{k}}^{(n)}\right)^{2} \\
\longrightarrow & \int_{0}^{1} \ldots \int_{0}^{1} Q\left(t_{u}, s_{1}, \ldots, s_{k}\right) \\
& \times Q\left(t_{v}, s_{1}, \ldots, s_{k}\right) d s_{1} \cdots d s_{k}, \quad \text { a.s.. }
\end{aligned}
$$

It turns into show that the difference

$$
\begin{aligned}
& \sum_{i_{1}, \ldots, i_{k}=1, i_{1} \neq \cdots \neq i_{k}}^{n} n^{2 k} \\
& \times\left(\int_{\left(\left(i_{1}-1\right) / n\right)}^{\left(i_{1} / n\right)} \cdots \int_{\left(\left(i_{k}-1\right) / n\right)}^{\left(i_{k} / n\right)} Q\left(t_{u}, s_{1}, \ldots, s_{k}\right) d s_{1} \cdots d s_{k}\right. \\
& \quad \times \int_{\left(\left(i_{1}-1\right) / n\right)}^{\left(i_{1} / n\right)} \cdots \int_{\left(\left(i_{k}-1\right) / n\right)}^{\left(i_{k} / n\right)} Q\left(t_{v}, s_{1}, \ldots, s_{k}\right) d s_{1} \cdots d s_{k} \\
& \quad-\int_{\left(\left(i_{1}-1\right) / n\right)}^{\left(i_{1} / n\right)} \cdots \int_{\left(\left(i_{k}-1\right) / n\right)}^{\left(i_{k} / n\right)} Q\left(t_{u}^{n}, s_{1}, \ldots, s_{k}\right) d s_{1} \cdots d s_{k}
\end{aligned}
$$

$$
\begin{aligned}
& \times \int_{\left(\left(i_{1}-1\right) / n\right)}^{\left(i_{1} / n\right)} \\
& \left.\cdots \int_{\left(\left(i_{k}-1\right) / n\right)}^{\left(i_{k} / n\right)} Q\left(t_{v}^{n}, s_{1}, \ldots, s_{k}\right) d s_{1} \cdots d s_{k}\right) \\
& \times\left(\xi_{i_{1}}^{(n)} \ldots \xi_{i_{k}}^{(n)}\right)^{2}
\end{aligned}
$$

tends to zero a.s. as $n$ tends to infinity.

Since $Q$ is increasing with respect to $t$ and $t_{u} \geq t_{u}^{n}, t_{v} \geq t_{v}^{n}$, we can get

$$
\begin{aligned}
& 0 \leq \sum_{i_{1}, \ldots, i_{k}=1, i_{1} \neq \cdots \neq i_{k}}^{n} n^{2 k} \\
& \times\left(\int_{\left(\left(i_{1}-1\right) / n\right)}^{\left(i_{1} / n\right)} \cdots \int_{\left(\left(i_{k}-1\right) / n\right)}^{\left(i_{k} / n\right)} Q\left(t_{u}, s_{1}, \ldots, s_{k}\right) d s_{1} \cdots d s_{k}\right. \\
& \times \int_{\left(\left(i_{1}-1\right) / n\right)}^{\left(i_{1} / n\right)} \cdots \int_{\left(\left(i_{k}-1\right) / n\right)}^{\left(i_{k} / n\right)} Q\left(t_{v}, s_{1}, \ldots, s_{k}\right) d s_{1} \cdots d s_{k} \\
& -\int_{\left(\left(i_{1}-1\right) / n\right)}^{\left(i_{1} / n\right)} \cdots \int_{\left(\left(i_{k}-1\right) / n\right)}^{\left(i_{k} / n\right)} Q\left(t_{u}^{n}, s_{1}, \ldots, s_{k}\right) d s_{1} \cdots d s_{k} \\
& \left.\times \int_{\left(\left(i_{1}-1\right) / n\right)}^{\left(i_{1} / n\right)} \cdots \int_{\left(\left(i_{k}-1\right) / n\right)}^{\left(i_{k} / n\right)} Q\left(t_{v}^{n}, s_{1}, \ldots, s_{k}\right) d s_{1} \cdots d s_{k}\right) \\
& \times\left(\xi_{i_{1}}^{(n)} \ldots \xi_{i_{k}}^{(n)}\right)^{2} \\
& \leq \sum_{i_{1}, \ldots, i_{k}=1, i_{1} \neq \cdots \neq i_{k}}^{n} n^{2 k} \int_{\left(\left(i_{1}-1\right) / n\right)}^{\left(i_{1} / n\right)} \\
& \cdots \int_{\left(\left(i_{k}-1\right) / n\right)}^{\left(i_{k} / n\right)} Q\left(t_{u}, s_{1}, \ldots, s_{k}\right) d s_{1} \cdots d s_{k}\left(\xi_{i_{1}}^{(n)} \cdots \xi_{i_{k}}^{(n)}\right)^{2} \\
& \times \int_{\left(\left(i_{1}-1\right) / n\right)}^{\left(i_{1} / n\right)} \\
& \cdots \int_{\left(\left(i_{k}-1\right) / n\right)}^{\left(i_{k} / n\right)}\left(Q\left(t_{v}, s_{1}, \ldots, s_{k}\right)-Q\left(t_{v}^{n}, s_{1}, \ldots, s_{k}\right)\right) \\
& \times d s_{1} \cdots d s_{k}
\end{aligned}
$$$$
+\sum_{i_{1}, \ldots, i_{k}=1, i_{1} \neq \cdots \neq i_{k}}^{n} n^{2 k}\left(\xi_{i_{1}}^{(n)} \ldots \xi_{i_{k}}^{(n)}\right)^{2}
$$$$
\times \int_{\left(\left(i_{1}-1\right) / n\right)}^{\left(i_{1} / n\right)}
$$$$
\cdots \int_{\left(\left(i_{k}-1\right) / n\right)}^{\left(i_{k} / n\right)}\left|Q\left(t_{v}^{n}, s_{1}, \ldots, s_{k}\right)-Q\left(t_{v}, s_{1}, \ldots, s_{k}\right)\right|
$$$$
\times d s_{1} \cdots d s_{k}
$$$$
\times \int_{\left(\left(i_{1}-1\right) / n\right)}^{\left(i_{1} / n\right)}
$$ 


$$
\begin{aligned}
& \quad \ldots \int_{\left.\left(i_{k}-1\right) / n\right)}^{\left(i_{k} / n\right)}\left|Q\left(t_{u}, s_{1}, \ldots, s_{k}\right)-Q\left(t_{u}^{n}, s_{1}, \ldots, s_{k}\right)\right| \\
& \quad \times d s_{1} \cdots d s_{k} \\
& \quad \sum_{i_{1}, \ldots, i_{k}=1, i_{1} \neq \cdots \neq i_{k}}^{n} n^{2 k} \int_{\left(\left(i_{1}-1\right) / n\right)}^{\left(i_{1} / n\right)} \\
& \quad \ldots \int_{\left(\left(i_{k}-1\right) / n\right)}^{\left(i_{k} / n\right)} Q\left(t_{v}, s_{1}, \ldots, s_{k}\right) d s_{1} \cdots d s_{k}\left(\xi_{i_{1}}^{n} \ldots \xi_{i_{k}}^{n}\right)^{2} \\
& \times \int_{\left(\left(i_{1}-1\right) / n\right)}^{\left(i_{1} / n\right)} \\
& \ldots \int_{\left(\left(i_{k}-1\right) / n\right)}^{\left(i_{k} / n\right)}\left|Q\left(t_{u}, s_{1}, \ldots, s_{k}\right)-Q\left(t_{u}^{n}, s_{1}, \ldots, s_{k}\right)\right| \\
& \quad \times d s_{1} \cdots d s_{k} \\
& \equiv \mathrm{I}+\mathrm{II}+\mathrm{III} .
\end{aligned}
$$

Notice that

$$
\begin{aligned}
& \int_{0}^{t_{u}} \int_{t_{v}^{n}}^{t_{v}} x^{k\left(H_{0}-(1 / 2)\right)} y^{k\left(H_{0}-(1 / 2)\right)} \\
& \quad \times\left[\int_{0}^{1} s^{1-2 H_{0}}(x-s)_{+}^{H_{0}-(3 / 2)}(y-s)_{+}^{H_{0}-(3 / 2)} d s\right]^{k} d x d y \\
& \quad \leq \int_{0}^{t_{u}} \int_{t_{v}^{n}}^{t_{v}}|x-y|^{\left(2 H_{0}-2\right) k} d x d y \\
& \quad=\int_{0}^{t_{u}} \int_{t_{v}^{n}}^{t_{v}}|x-y|^{2 H-2} d x d y \\
& \leq \int_{0}^{1} \int_{0}^{1}|x-y|^{2 H-2} I_{\left(0, t_{u}\right]}(x) I_{\left[\left[n t_{v}\right] / n, t_{v}\right]}(y) d x d y
\end{aligned}
$$

$t_{u} \geq t_{u}^{n}, t_{v} \geq t_{v}^{n}$. We can get, by using the first part of this proof,

$$
\begin{aligned}
& I \longrightarrow \int_{0}^{1} \ldots \int_{0}^{1} Q\left(t_{u}, s_{1}, \ldots, s_{k}\right) \\
& \times\left(Q\left(t_{v}, s_{1}, \ldots, s_{k}\right)-Q\left(t_{v}^{n}, s_{1}, \ldots, s_{k}\right)\right) \\
& \times d s_{1} \cdots d s_{k} \\
& \leq C \int_{[0,1]^{k}}( \prod_{j=1}^{k} s_{j}^{1-2 H_{0}} \int_{0}^{t_{u}} \int_{t_{v}^{n}}^{t_{v}} x^{k\left(H_{0}-(1 / 2)\right)} y^{k\left(H_{0}-(1 / 2)\right)} \\
&\left.\times \prod_{j=1}^{k}\left(x-s_{j}\right)_{+}^{H_{0}-(3 / 2)}\left(y-s_{j}\right)_{+}^{H_{0}-(3 / 2)} d x d y\right) \\
& \times d s_{1} \cdots d s_{k}
\end{aligned}
$$

$$
\begin{gathered}
=C \int_{0}^{t_{u}} \int_{t_{v}^{n}}^{t_{v}} x^{k\left(H_{0}-(1 / 2)\right)} y^{k\left(H_{0}-(1 / 2)\right)} \\
\times\left[\int_{[0,1]^{k}} \prod_{j=1}^{k} s_{j}^{1-2 H_{0}}\left(x-s_{j}\right)_{+}^{H_{0}-(3 / 2)}\right. \\
\left.\times\left(y-s_{j}\right)_{+}^{H_{0}-(3 / 2)} d s_{1} \cdots d s_{k}\right] d x d y \\
=C \int_{0}^{t_{u}} \int_{t_{v}^{n}}^{t_{v}} x^{k\left(H_{0}-(1 / 2)\right)} y^{k\left(H_{0}-(1 / 2)\right)} \\
\times\left[\int_{0}^{1} s^{1-2 H_{0}}(x-s)_{+}^{H_{0}-(3 / 2)}(y-s)_{+}^{H_{0}-(3 / 2)} d s\right]^{k} \\
\times d x d y \longrightarrow 0
\end{gathered}
$$

as $n$ tends to infinity because $0 \leq t_{v}-\left[n t_{v}\right] / n \leq 1 / n$. Similarly the last two summands II and III also tend to zero and we have that when $n \rightarrow \infty$,

$$
\begin{aligned}
& \sum_{i_{1}, \ldots, i_{k}=1, i_{1} \neq \ldots \neq i_{k}}^{n} n^{2 k} \int_{\left(\left(i_{1}-1\right) / n\right)}^{\left(i_{1} / n\right)} \\
& \ldots \int_{\left(\left(i_{k}-1\right) / n\right)}^{\left(i_{k} / n\right)} Q\left(\frac{\left[n t_{u}\right]}{n}, s_{1}, \ldots, s_{k}\right) d s_{1} \cdots d s_{k} \\
\times & \int_{\left(\left(i_{1}-1\right) / n\right)}^{\left(i_{1} / n\right)} \cdots \int_{\left(\left(i_{k}-1\right) / n\right)}^{\left(i_{k} / n\right)} Q\left(\frac{\left[n t_{v}\right]}{n}, s_{1}, \ldots, s_{k}\right) d s_{1} \cdots d s_{k} \\
\times & \left(\xi_{i_{1}}^{(n)} \ldots \xi_{i_{k}}^{(n)}\right)^{2} \\
\longrightarrow & \int_{0}^{1} \ldots \int_{0}^{1} Q\left(t_{u}, s_{1}, \ldots, s_{k}\right) Q\left(t_{v}, s_{1}, \ldots, s_{k}\right) d s_{1} \cdots d s_{k}
\end{aligned}
$$

holds a.s., and the lemma follows.

We can prove the main result in this paper.

Proof of Theorem 2. We prove this theorem by using Theorem 4 . We first have to prove that the finite-dimensional distributions of $Z^{n}$ converge to those of $Z_{H, k}$.

For any $a_{1}, \ldots, a_{d} \in \mathbb{R}$ and $t_{1}, \ldots, t_{d} \in[0,1]$, we want to show that the the linear combination

$$
Y^{n}:=\sum_{u=1}^{d} a_{u} Z_{t_{u}}^{n}
$$

converges in distribution to a normally distributed random variable with expectation zero and variance

$$
E\left(\sum_{u=1}^{d} a_{u} Z_{t_{u}}\right)^{2}
$$


The fact that the expectation is zero is trivial. Let us write $Y^{n}$ as

$$
\begin{aligned}
Y^{n}= & \sum_{i_{1}, \ldots, i_{k}=1, i_{1} \neq \cdots \neq i_{k}}^{n} n^{k}\left(\xi_{i_{1}}^{(n)} \ldots \xi_{i_{k}}^{(n)}\right)^{2} \\
& \times \sum_{u=1}^{d} a_{u} \int_{\left(\left(i_{1}-1\right) / n\right)}^{\left(i_{1} / n\right)} \\
& \cdots \int_{\left(\left(i_{k}-1\right) / n\right)}^{\left(i_{k} / n\right)} Q\left(\frac{[n t]}{n}, s_{1}, \ldots, s_{k}\right) d s_{1} \cdots d s_{k} \\
:= & \sum_{i_{1}, \ldots, i_{k}=1, i_{1} \neq \cdots \neq i_{k}}^{n} Y_{i_{1}, \ldots, i_{k}}^{n} .
\end{aligned}
$$
have

The Lindeberg condition is satisfied if for any $\varepsilon>0$, we

$$
\sum_{i_{1}, \ldots, i_{k}=1, i_{1} \neq \cdots \neq i_{k}}^{n} E\left[\left(Y_{i_{1}, \ldots, i_{k}}^{n}\right)^{2} I_{\left\{\left|Y_{i_{1}, \ldots, i_{k}}^{n}\right|>\varepsilon\right\}} \mid \mathscr{F}_{i_{1}-1, \ldots, i_{k}-1}^{n}\right] \stackrel{p}{\rightarrow} 0,
$$

as $n$ tends to infinity. Consider the set

$$
\left\{\left|Y_{i_{1}, \ldots, i_{k}}^{n}\right|>\varepsilon\right\}=\left\{\left(Y_{i_{1}, \ldots, i_{k}}^{n}\right)^{2}>\varepsilon^{2}\right\}
$$

We get an upper bound to $Y_{i_{1}, \ldots, i_{k}}^{n}$ by noticing that $\mathrm{Q}\left(t, s_{1}, \ldots, s_{k}\right)$ is increasing with respect to $t$ and decreasing with respect to $s_{1}, \ldots, s_{k}$.

Consider the following:

$$
\begin{aligned}
& \left(Y_{i_{1}, \ldots, i_{k}}^{n}\right)^{2} \\
& =\left(n^{k}\left(\xi_{i_{1}}^{(n)} \ldots \xi_{i_{k}}^{(n)}\right)^{2} \sum_{u=1}^{d} a_{u} \int_{\left(\left(i_{1}-1\right) / n\right)}^{\left(i_{1} / n\right)}\right. \\
& \left.\ldots \int_{\left(\left(i_{k}-1\right) / n\right)}^{\left(i_{k} / n\right)} Q\left(\frac{[n t]}{n}, s_{1}, \ldots, s_{k}\right) d s_{1} \cdots d s_{k}\right)^{2} \\
& \leq n^{2 k}\left(\xi_{i_{1}}^{(n)} \ldots \xi_{i_{k}}^{(n)}\right)^{2}\left(\sum_{u=1}^{d}\left|a_{u}\right|\right)^{2} \\
& \quad \times\left(\int_{\left(\left(i_{1}-1\right) / n\right)}^{\left(i_{1} / n\right)} \ldots \int_{\left.\left(i_{k}-1\right) / n\right)}^{\left(i_{k} / n\right)} Q\left(1, s_{1}, \ldots, s_{k}\right) d s_{1} \cdots d s_{k}\right)^{2} \\
& \leq n^{k}\left(\xi_{i_{1}}^{(n)} \ldots \xi_{i_{k}}^{(n)}\right)^{2}\left(\sum_{u=1}^{d}\left|a_{u}\right|\right)^{2} \\
& \quad \times \int_{0}^{(1 / n)} \ldots \int_{0}^{(1 / n)} Q Q^{2}\left(1, s_{1}, \ldots, s_{k}\right) d s_{1} \ldots d s_{k} \\
& =: n^{k}\left(\xi_{i_{1}}^{(n)} \ldots \xi_{i_{k}}^{(n)}\right)^{2} A \delta^{n},
\end{aligned}
$$

where $A:=\left(\sum_{u=1}^{d}\left|a_{u}\right|\right)^{2}$ and $\delta^{n}:=\int_{0}^{1 / n} \cdots \int_{0}^{1 / n} Q^{2}\left(1, s_{1}\right.$, $\left.\ldots, s_{k}\right) d s_{1} \ldots d s_{k}$. Thus we obtain

$$
\left\{\left|Y_{i_{1}, \ldots, i_{k}}^{n}\right|>\varepsilon\right\} \subset\left\{n^{k}\left(\xi_{i_{1}}^{(n)} \ldots \xi_{i_{k}}^{(n)}\right)^{2} A \delta^{n}>\varepsilon^{2}\right\} .
$$

Combining this with the Cauchy-Schwartz inequality, we can get that

$$
\begin{aligned}
& E\left[\left(Y_{i_{1}, \ldots, i_{k}}^{n}\right)^{2} I_{\left\{\left|Y_{i_{1}, \ldots, i_{k}}^{n}\right|>\varepsilon\right\}} \mid \mathscr{F}_{i_{1}-1, \ldots, i_{k}-1}^{n}\right] \\
& =\left(n^{k} \sum_{u=1}^{d} a_{u} \int_{\left(\left(i_{1}-1\right) / n\right)}^{\left(i_{1} / n\right)}\right. \\
& \left.\cdots \int_{\left(\left(i_{k}-1\right) / n\right)}^{\left(i_{k} / n\right)} Q\left(\frac{[n t]}{n}, s_{1}, \ldots, s_{k}\right) d s_{1} \cdots d s_{k}\right)^{2} \\
& \times E\left[\left(\xi_{i_{1}}^{(n)} \ldots \xi_{i_{k}}^{(n)}\right)^{2} I_{\left\{\left|Y_{i_{1}, \ldots, i_{k}}^{n}\right|>\varepsilon\right\}} \mid \mathscr{F}_{i_{1}-1, \ldots, i_{k}-1}^{n}\right] \\
& \leq\left(n^{k} \sum_{u=1}^{d} a_{u} \int_{0}^{1 / n} \cdots \int_{0}^{1 / n} Q\left(1, s_{1}, \ldots, s_{k}\right) d s_{1} \cdots d s_{k}\right)^{2} \\
& \times\left(\frac{C^{2}}{n}\right)^{k} E\left[I_{\left\{\left|Y_{i_{1}, \ldots, i_{k}}^{n}\right|>\varepsilon\right\}} \mid \mathscr{F}_{i_{1}-1, \ldots, i_{k}-1}^{n}\right] \\
& \leq C^{2 k} A \delta^{n} E\left[I_{\left\{n^{k}\left(\xi_{i_{1}}^{(n)} \ldots \xi_{i_{k}}^{(n)}\right)^{2} A \delta^{n}>\varepsilon^{2}\right\}} \mid \mathscr{F}_{i_{1}-1, \ldots, i_{k}-1}^{n}\right],
\end{aligned}
$$

a.s., which deduces that the Lindeberg condition is satisfied:

$$
\begin{aligned}
& \sum_{i_{1}, \ldots, i_{k}=1, i_{1} \neq \cdots \neq i_{k}}^{n} E\left[\left(Y_{i_{1}, \ldots, i_{k}}^{n}\right)^{2} I_{\left\{\left|Y_{i_{1}, \ldots, i_{k}}^{n}\right|>\varepsilon\right\}} \mid \mathscr{F}_{i_{1}-1, \ldots, i_{k}-1}^{n}\right] \\
& \leq \sum_{i_{1}, \ldots, i_{k}=1, i_{1} \neq \cdots \neq i_{k}}^{n} C^{2 k} A \delta^{n} E\left[I_{\left\{n^{k}\left(\xi_{i_{1}}^{(n)} \ldots \xi_{i_{k}}^{(n)}\right)^{2} A \delta^{n}>\varepsilon^{2}\right\}} \mid \mathscr{F}_{i_{1}-1, \ldots, i_{k}-1}^{n}\right] \\
& \leq C^{2 k} A \delta^{n} \sum_{i_{1}, \ldots, i_{k}=1, i_{1} \neq \cdots \neq i_{k}}^{n} I_{\left\{C^{2 k} A \delta^{n}>\varepsilon^{2}\right\}} \longrightarrow 0,
\end{aligned}
$$

as $n$ tends to infinity, because of $\delta^{n} \rightarrow 0$ and $I_{\left\{C^{2 k} A \delta^{n}>\varepsilon^{2}\right\}}=0$ for large $n$. Moreover, we have

$$
\begin{aligned}
& \quad \sum_{i_{1}, \ldots, i_{k}=1, i_{1} \neq \cdots \neq i_{k}}^{n}\left(Y_{i_{1}, \ldots, i_{k}}^{n}\right)^{2} \\
& =\sum_{u=1, v=1}^{d} a_{u} a_{v} \sum_{i_{1}, \ldots i_{k}=1, i_{1} \neq \cdots \neq i_{k}}^{n} n^{2 k} \int_{\left(\left(i_{1}-1\right) / n\right)}^{\left(i_{1} / n\right)} \\
& \quad \ldots \int_{\left(\left(i_{k}-1\right) / n\right)}^{\left(i_{k} / n\right)} Q\left(\frac{\left[n t_{u}\right]}{n}, s_{1}, \ldots, s_{k}\right) d s_{1} \cdots d s_{k} \\
& \quad \times \int_{\left.\left(i_{1}-1\right) / n\right)}^{\left(i_{1} / n\right)} \cdots \int_{\left.\left(i_{k}-1\right) / n\right)}^{\left(i_{k} / n\right)} Q\left(\frac{\left[n t_{v}\right]}{n}, s_{1}, \ldots, s_{k}\right) d s_{1} \cdots d s_{k} \\
& \quad \times\left(\xi_{i_{1}}^{(n)} \ldots \xi_{i_{k}}^{(n)}\right)^{2},
\end{aligned}
$$


for all $n \geq 1$. By Lemma 6 and the fact that

$$
\begin{aligned}
E Z_{t_{u}} Z_{t_{v}}= & \int_{0}^{\min \left(t_{u}, t_{v}\right)} \\
& \cdots \int_{0}^{\min \left(t_{u}, t_{v}\right)} Q\left(t_{u}, s_{1}, \ldots, s_{k}\right) Q\left(t_{v}, s_{1}, \ldots, s_{k}\right) \\
& \quad \times d s_{1} \ldots d s_{k},
\end{aligned}
$$

it follows that the sum $\sum_{i_{1}, \ldots, i_{k}=1, i_{1} \neq \cdots \neq i_{k}}^{n}\left(Y_{i_{1}, \ldots, i_{k}}^{n}\right)^{2}$ converges to

$$
\begin{aligned}
& \sum_{u=1, v=1}^{d} a_{u} a_{v} \int_{0}^{1} \\
& \quad \ldots \int_{0}^{1} Q\left(t_{u}, s_{1}, \ldots, s_{k}\right) Q\left(t_{v}, s_{1}, \ldots, s_{k}\right) d s_{1} \ldots d s_{k} \\
& =E\left(\sum_{u=1}^{d} a_{u} Z_{t_{u}}\right)^{2} .
\end{aligned}
$$

So by Theorem 5 , the finite-dimensional distributions of $Z^{n}$ converge to those of $Z_{H, k}$.

Finally, we need to prove the tightness of the sequence $Z^{n}$ in $D$. Let $s, t \in[0,1], s<t$. It is easy to prove that

$$
E\left[\xi_{i_{1}}^{(n)} \xi_{j_{1}}^{(n)} \cdots \xi_{i_{k}}^{(n)} \xi_{j_{k}}^{(n)}\right]=0
$$

provided $i_{l} \neq j_{l}$ for some $l=1,2, \ldots, k$. It follows from the Cauchy-Schwartz inequality that

$$
\begin{aligned}
& E\left(Z_{t}^{n}-Z_{s}^{n}\right)^{2} \\
& =E\left(\sum_{i_{1}, \ldots, i_{k}=1, i_{1} \neq \cdots \neq i_{k}}^{[n t]} n^{k} \int_{\left(\left(i_{1}-1\right) / n\right)}^{\left(i_{1} / n\right)} \ldots \int_{\left(\left(i_{k}-1\right) / n\right)}^{\left(i_{k} / n\right)} \xi_{i_{1}}^{(n)} \ldots \xi_{i_{k}}^{(n)}\right. \\
& \times\left[Q\left(\frac{[n t]}{n}, s_{1}, \ldots, s_{k}\right)\right. \\
& \left.\left.\left.=\quad \sum_{i_{1}, \ldots, i_{k}=1, i_{1} \neq \ldots \neq i_{k}}^{[n t]}-Q\left(\frac{[n s]}{n}, s_{1}, \ldots, s_{k}\right)\right] d s_{1} \cdots d s_{k}\right)^{2} \ldots \xi_{i_{k}}^{(n)}\right)^{2} \\
& \left.\times\left(n^{k} \int_{\left(\left(i_{1}-1\right) / n\right)}^{\left(i_{1} / n\right)}-Q\left(\frac{[n s]}{n}, s_{1}, \ldots, s_{k}\right)\right] d s_{1} \cdots d s_{k}\right)^{2} \\
& \ldots \int_{\left(\left(i_{k}-1\right) / n\right)}^{\left(i_{k} / n\right)}\left[Q\left(\frac{[n t]}{n}, s_{1}, \ldots, s_{k}\right)\right.
\end{aligned}
$$

$$
\begin{aligned}
& \leq \sum_{i_{1}, \ldots, i_{k}=1, i_{1} \neq \cdots \neq i_{k}}^{[n t]} n^{k} E\left(\xi_{i_{1}}^{(n)} \ldots \xi_{i_{k}}^{(n)}\right)^{2} \\
& \times \int_{\left(\left(i_{1}-1\right) / n\right)}^{\left(i_{1} / n\right)} \\
& \cdots \int_{\left(\left(i_{k}-1\right) / n\right)}^{\left(i_{k} / n\right)}\left[Q\left(\frac{[n t]}{n}, s_{1}, \ldots, s_{k}\right)\right. \\
& \left.\quad-Q\left(\frac{[n s]}{n}, s_{1}, \ldots, s_{k}\right)\right]^{2} d s_{1} \cdots d s_{k}
\end{aligned}
$$

$$
\begin{aligned}
& \leq \sum_{i_{1}, \ldots, i_{k}=1, i_{1} \neq \cdots \neq i_{k}}^{[n t]} C^{2 k} \\
& \times \int_{\left(\left(i_{1}-1\right) / n\right)}^{\left(i_{1} / n\right)}\left[Q\left(\frac{[n t]}{n}, s_{1}, \ldots, s_{k}\right)\right. \\
& \ldots \int_{\left(\left(i_{k}-1\right) / n\right)}^{\left(i_{k} / n\right)}\left[Q\left(\frac{[n s]}{n}, s_{1}, \ldots, s_{k}\right)\right]^{2} d s_{1} \cdots d s_{k} \\
& \leq C^{2 k} \int_{0}^{1} \ldots \int_{0}^{1}\left[Q\left(\frac{[n t]}{n}, s_{1}, \ldots, s_{k}\right)\right. \\
& \left.\leq C^{2 k \mid} \frac{[n t]}{n}-\left.\frac{[n s]}{n}\right|^{2 H} \cdot Q\left(\frac{[n s]}{n}, s_{1}, \ldots, s_{k}\right)\right]^{2} d s_{1} \cdots d s_{k}
\end{aligned}
$$

Notice that for any $s<t<r$,

$$
\begin{aligned}
E\left|Z_{t}^{n}-Z_{s}^{n}\right|\left|Z_{r}^{n}-Z_{t}^{n}\right| & \leq\left(E\left(Z_{t}^{n}-Z_{s}^{n}\right)^{2}\right)^{1 / 2}\left(E\left(Z_{r}^{n}-Z_{t}^{n}\right)^{2}\right)^{1 / 2} \\
& \leq C^{2 k}\left|\frac{[n t]}{n}-\frac{[n s]}{n}\right|^{H}\left|\frac{[n r]}{n}-\frac{[n t]}{n}\right|^{H} \\
& \leq C^{2 k \mid}\left|\frac{[n r]}{n}-\frac{[n s]}{n}\right|^{2 H}
\end{aligned}
$$

We see that

$$
E\left|Z_{t}^{n}-Z_{s}^{n}\right|\left|Z_{r}^{n}-Z_{t}^{n}\right| \leq C^{2 k}|r-s|^{2 H},
$$

if $r-s \geq 1 / n$. On the other hand if $r-s<1 / n$, then either $s$ and $t$ or $t$ and $r$ belong to a same subinterval $[m / n,(m+1) / n]$ for some integer $m$. Thus the left-hand side of (67) is zero. Therefore, (67) holds for all $s<t<r$ and $Z^{n} \stackrel{d}{\rightarrow} Z_{H, k}$ by Theorem 4 . This completes the proof.

\section{Conflict of Interests}

The authors declare that there is no conflict of interests regarding the publication of this paper. 


\section{Acknowledgments}

The authors would like to express their sincere gratitude to the editor and the anonymous referees for their valuable comments and error corrections. The project is sponsored by Natural Science Foundation of Anhui Province (no. 1408085QA10), NSFC (11171062), and Innovation Program of Shanghai Municipal Education Commission (12ZZ063).

\section{References}

[1] M. S. Taqqu, "A bibliographical guide to self-similar processes and long-range dependence," in Dependence in Probability and Statistics, pp. 137-162, Birkhäuser, Boston, Mass, USA, 1986.

[2] P. Embrechts and M. Maejima, Self-Similar Processes, Princeton University Press.

[3] G. Samorodnitsky and M. Taqqu, Stable Non-Gaussian Random Variables, Chapman and Hall, London, UK, 1994.

[4] R. L. Dobrushin and P. Major, "Non-central limit theorems for nonlinear functionals of Gaussian fields," Zeitschrift für Wahrscheinlichkeitstheorie und Verwandte Gebiete, vol. 50, no. 1, pp. 27-52, 1979.

[5] M. S. Taqqu, "Convergence of integrated processes of arbitrary Hermite rank," Zeitschrift für Wahrscheinlichkeitstheorie und Verwandte Gebiete, vol. 50, no. 1, pp. 53-83, 1979.

[6] M. S. Taqqu, "Weak convergence to fractional Brownian motion and to the Rosenblatt process," vol. 31, pp. 287-302, 1975.

[7] J. Bardet and C. A. Tudor, "A wavelet analysis of the Rosenblatt process: chaos expansion and estimation of the self-similarity parameter," Stochastic Processes and Their Applications, vol. 120, no. 12, pp. 2331-2362, 2010.

[8] A. Chronopoulou, F. G. Viens, and C. A. Tudor, "Variations and Hurst index estimation for a Rosenblatt process using longer filters," Electronic Journal of Statistics, vol. 3, pp. 1393-1435, 2009.

[9] M. Li, "A class of negatively fractal dimensional Gaussian random functions," Mathematical Problems in Engineering, vol. 2011, Article ID 291028, 18 pages, 2011.

[10] M. Li, "Power spectrum of generalized fractional Gaussian noise," Advances in Mathematical Physics, vol. 2013, Article ID 315979, 3 pages, 2013.

[11] M. Li and S. C. Lim, "Power spectrum of generalized Cauchy process," Telecommunication Systems, vol. 43, no. 3-4, pp. 219222, 2010.

[12] M. H. Heydari, M. R. Hooshmandasl, F. Maalek Ghaini, and C. Cattani, "A computational method for solving stochastic Itô-Volterra integral equations based on stochastic operational matrix for generalized hat basis functions," Journal of Computational Physics, vol. 270, pp. 402-415, 2014.

[13] M. H. Heydari, M. R. Hooshmandasl, F. Mohammadi, and C. Cattani, "Wavelets method for solving systems of nonlinear singular fractional Volterra integro-differential equations," Communications in Nonlinear Science and Numerical Simulation, vol. 19, no. 1, pp. 37-48, 2014.

[14] M. Maejima and C. A. Tudor, "Wiener integrals with respect to the Hermite process and a non-central limit theorem," Stochastic Analysis and Applications, vol. 25, no. 5, pp. 10431056, 2007.

[15] V. Pipiras and M. S. Taqqu, "Regularization and integral representations of Hermite processes," Statistics \& Probability Letters, vol. 80, no. 23-24, pp. 2014-2023, 2010.
[16] S. Torres and C. A. Tudor, "Donsker type theorem for the Rosenblatt process and a binary market model," Stochastic Analysis and Applications, vol. 27, no. 3, pp. 555-573, 2009.

[17] C. A. Tudor, "Analysis of the Rosenblatt process," ESAIM: Probability and Statistics, vol. 12, pp. 230-257, 2008.

[18] C. A. Tudor and F. G. Viens, "Variations and estimators for self-similarity parameters via Malliavin calculus," The Annals of Probability, vol. 37, no. 6, pp. 2093-2134, 2009.

[19] P. Hall, W. Hardle, and T. Kleinow, "Semiparametric bootstrap approach to hypothesis tests and confidence intervals for the Hurst coefficient," Statistical Inference for Stochastic Processes, vol. 3, no. 3, pp. 263-276, 2000.

[20] N. N. Leonenko and W. A. Woyczynski, "Scaling limits of solutions of the heat equation for singular non-Gaussian data," Journal of Statistical Physics, vol. 91, no. 1-2, pp. 423-438, 1998.

[21] E. Alòs, O. Mazet, and D. Nualart, "Stochastic calculus with respect to Gaussian processes," The Annals of Probability, vol. 29, no. 2, pp. 766-801, 2001.

[22] F. Biagini, Y. Hu, B. Âksendal, and T. Zhang, Stochastic Calculus for Fractional Brownian Motion and Applications, Probability and Its Application, Springer, Berlin, Germany, 2008.

[23] Y. Hu, "Integral transformations and anticipative calculus for fractional Brownian motions," Memoirs of the American Mathematical Society, vol. 175, no. 825, 2005.

[24] Y. S. Mishura, Stochastic Calculus for Fractional Brownian Motion and Related Processes, vol. 1929 of Lecture Notes in Mathematics, Springer, Berlin, Germany, 2008.

[25] A. Nieminen, "Fractional Brownian motion and martingaledifferences," Statistics \& Probability Letters, vol. 70, no. 1, pp. 110, 2004.

[26] D. Nualart, The Malliavin Calculus and Related Topics, Springer, 2006.

[27] G. Peccati and M. S. Taqqu, Wiener Chaos: Moments, Cumulants and Diagrams, Springer, 2011.

[28] P. Billingsley, Convergence of Probability Measures, Wiley, New York, NY, USA, 1968.

[29] P. Hall and C. C. Heyde, Martingale Limit Theory and Its Application, Academic Press, 1980. 


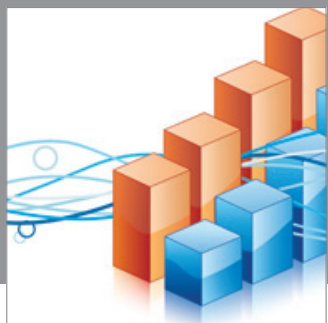

Advances in

Operations Research

mansans

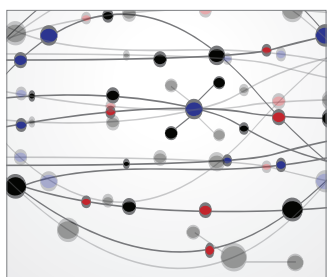

The Scientific World Journal
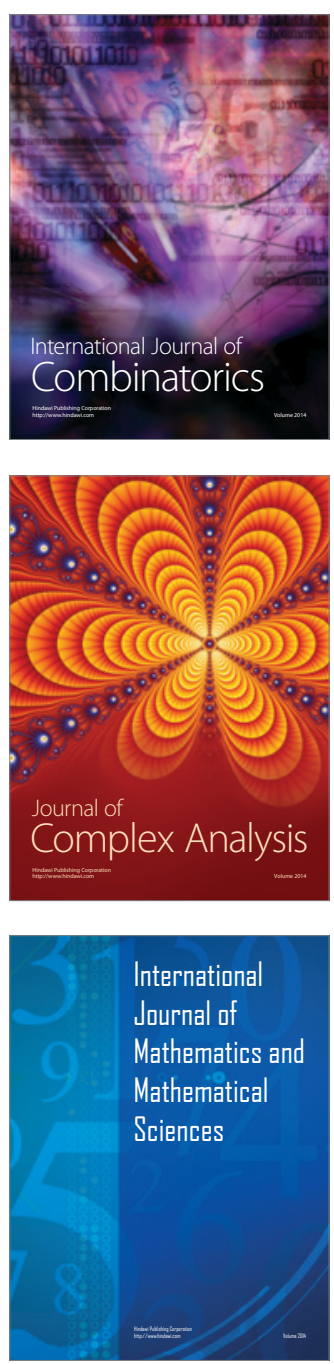
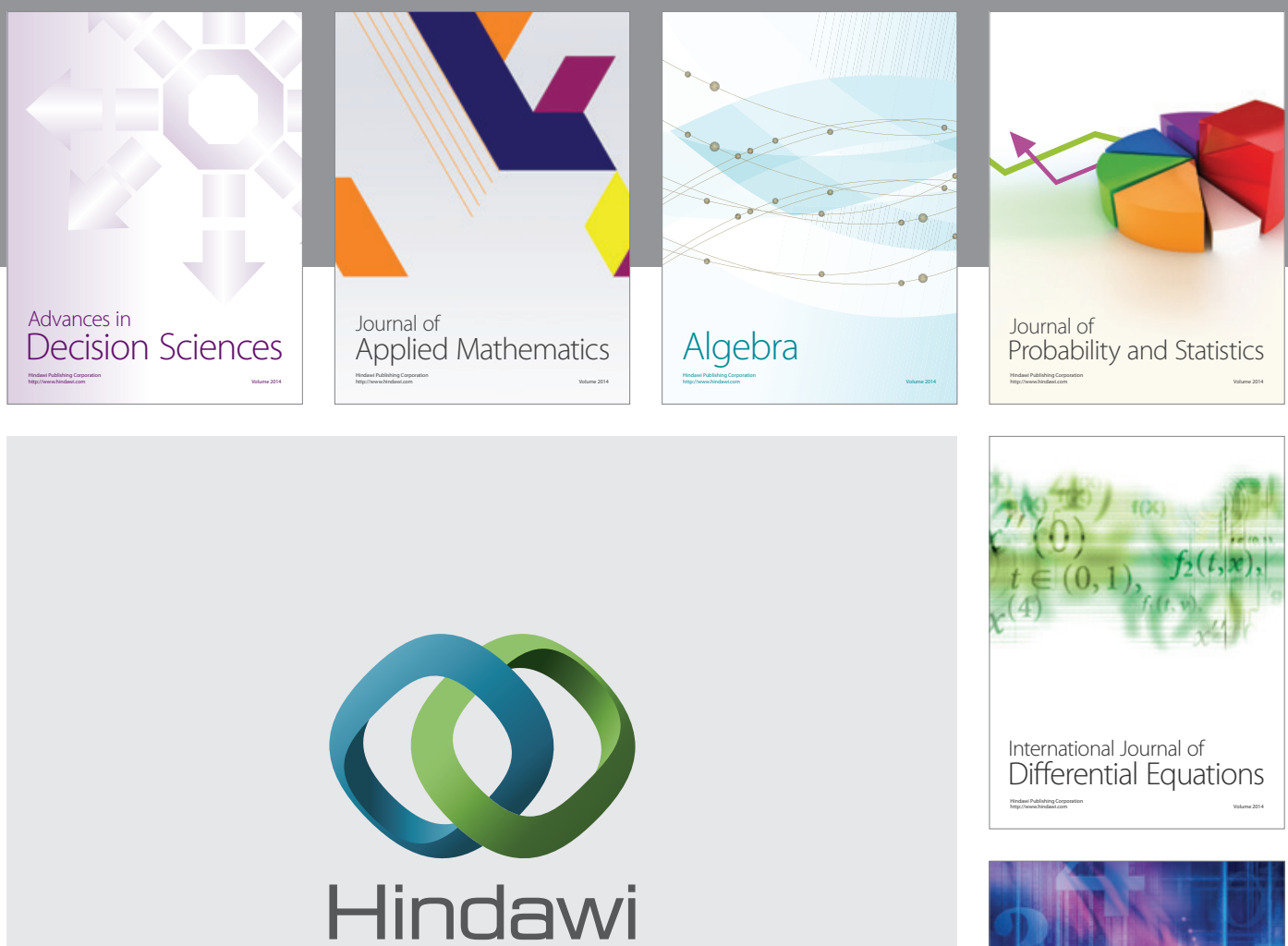

Submit your manuscripts at http://www.hindawi.com
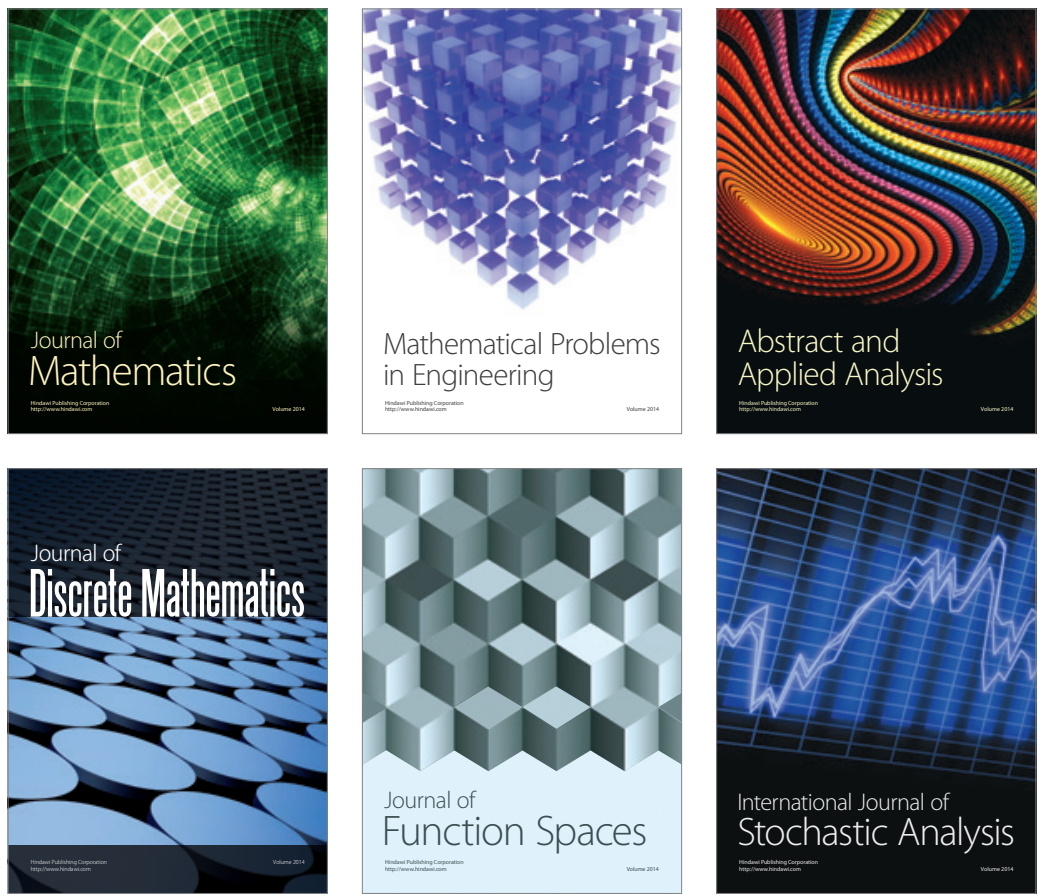

Journal of

Function Spaces

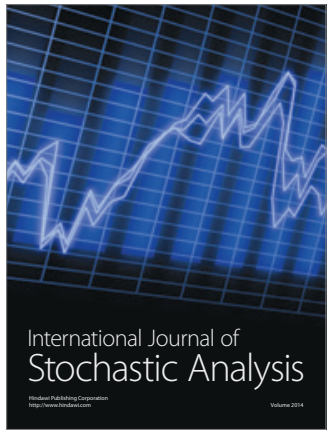

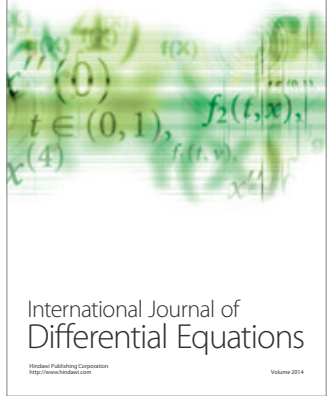
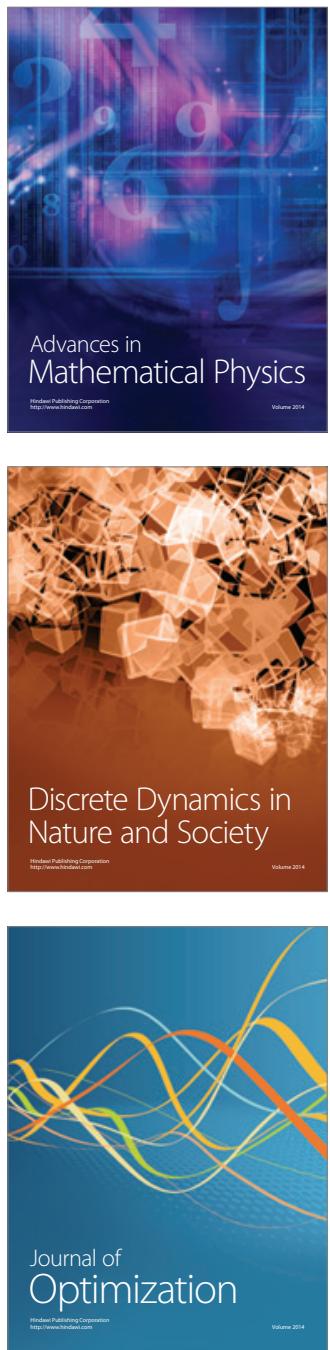